\title{
REAÇÃO DE FIGUEIRAS A TRÊS ESPÉCIES DE NEMATOIDES-DAS-GALHAS ${ }^{1}$
}

\author{
MARYLIA GABRIELLA SILVA COSTA², ÉRIKA CRISTINA SOUZA DA SILVA CORREIA ${ }^{3}$, \\ LUIS LESSI DOS REIS ${ }^{4}$, SILVIA RENATA SICILIANO WILCKEN 5
}

RESUMO- Os nematoides-das-galhas são considerados os patógenos que habitam o solo de maior importância na cultura da figueira no mundo. O objetivo do presente estudo foi avaliar a reação de genótipos de figueira a Meloidogyne javanica, M. incognita e M. enterolobii, com a possibilidade de serem utilizados como porta-enxerto resistente a esse patógeno. Os genótipos foram inoculados com 5.000 ovos e eventuais juvenis de segundo estádio das espécies dos nematoides em teste. As avaliações foram feitas aos 120 dias após a inoculação. As variáveis avaliadas foram: os índices de galhas, de massas de ovos e o fator de reprodução do nematoide. Todos os genótipos estudados comportaram-se como suscetíveis a M. javanica, M. incognita e M. enterolobii.

Termos para indexação: Ficus carica L., resistência, Meloidogyne.

\section{REACTION OF FIG TREE TO THREE SPECIES OF ROOT-KNOT NEMATODES}

\begin{abstract}
Root-knot nematodes are the main soil pathogens of fig culture around the world. The aim of this study was to evaluate the reaction of fig genotypes to $M$. javanica, M. incognita and M. enterolobii, for using as rootstocks. Plants were inoculated with 5,000 eggs of the nematode test. Gall index, egg mass index and reproduction factor were evaluated at 120 days after inoculation. All genotypes were susceptible to M. javanica, M. incognita and M. enterolobii.
\end{abstract}

Index terms: Ficus carica L., resistance, Meloidogyne.

\section{INTRODUÇÃO}

A figueira é originária da Ásia Menor e da Síria, situadas na região mediterrânea (LEONEL, 2008). Pertence à família Moraceae, que é composta por cerca de 60 gêneros, com mais de 2.000 espécies. O maior gênero da família é o Ficus, com cerca de 750 espécies. A espécie de maior importância no Brasil é o figo (Ficus carica L.) (PIO et al., 2011).

Ficus carica é produzido e consumido em todo o mundo. A produção dessa espécie é destinada tanto para comercialização in natura quanto para industrialização (SILVA et al., 2011). O Brasil é o oitavo maior produtor mundial, com uma produção de 28.010 toneladas (FAO, 2012). Os cultivos comerciais de figo neste País baseiam-se praticamente na plantação de uma única variedade, a 'Roxo de Valinhos', caracterizada pela sua rusticidade, vigor e excelente produção de frutos (PENTEADO, 1999).

Dentre os nematoides encontrados em áreas de cultivo de figo, Meloidogyne spp. são os mais frequentes e provavelmente os causadores dos maiores danos (EL-BORAI; DUNCAN, 2005). Foi relatado pela primeira vez em 1889, nos Estados Unidos da América, e a partir daí, tem sido observada a ocorrência em várias regiões do mundo, mostrando-se limitante a essa cultura (NEAL, 1889; MCSORLEY, 1992; ABRANTES et al., 2008). $\mathrm{Na}$ África do Sul, foi o gênero mais frequente em levantamentos conduzidos em diferentes regiões produtoras de figo e considerado um dos fatores mais

\footnotetext{
${ }^{1}$ (Trabalho 168-14). Recebido em: 09-05-2014. Aceito para publicação em: 27-01-2015.

${ }^{2}$ Mestranda em Proteção de Plantas na Faculdade de Ciências Agronômicas, FCA/UNESP, Câmpus de Botucatu-SP. E-mail: marylia gabriella@hotmail.com

${ }^{3}$ Doutoranda em Proteção de Plantas na Faculdade de Ciências Agronômicas, FCA/UNESP, Câmpus de Botucatu-SP. E-mail: erikacristina correia@hotmail.com

${ }^{4}$ Professor do ensino, básico técnico e tecnológico do Instituto Federal de Educação, Ciência e Tecnologia de Mato Grosso - IFMT/ Câmpus Confresa-MT. E-mail: lessireis@yahoo.com.br

${ }^{5}$ Professora Assistente Doutora na UNESP/FCA - Faculdade de Ciências Agronômicas - Câmpus de Botucatu-SP. E-mail: srenata@ fca.unesp.br
} 
limitantes para a cultura. As espécies encontradas foram Meloidogyne javanica e M. incognita. Houve também associação entre Heterodera fici e $M$. incognita resultando em perda completa da colheita de figo (WOHLFARTER et al., 2011).

No Brasil, Meloidogyne incognita (Kofoid e White) Chitwood é considerada a principal espécie de nematoide na cultura do figo, sendo encontrada com frequência nos pomares dessa cultura (CAMPOS, 1997). Foi detectada em $80 \%$ das amostras retiradas de pomares de figo do Rio Grande do Sul e de São Paulo (LIMA-MEDINA et al., 2006). Também foi relatada nos pomares dessa frutífera, na região noroeste do Paraná (DIAS-ARIEIRA et al., 2010). Em estudo recente, foi constada sua disseminação para os demais pomares do Rio Grande do Sul, de forma que foi encontrada em $100 \%$ das amostras, além de sua associação com Meloidogyne javanica (Treub) Chitwood (LIMA-MEDINA et al., 2013).

O controle de nematoides parasitos de plantas, de maneira geral, é difícil de ser realizado. Em culturas perenes, como é o caso da figueira, tornase ainda mais problemático. Uma das poucas opções seria o controle químico; entretanto, no Brasil, não há registro de nematicidas para esta cultura (AGROFIT, 2014). Uma alternativa de controle seria a utilização de variedades resistentes. Sendo assim, o objetivo do trabalho foi avaliar a reação de genótipos de figo a Meloidogyne enterolobii Yang e Eisenback, M. javanica e M. incognita, visando à busca de resistência a estes nematoides.

\section{MATERIAL E MÉTODOS}

Três experimentos foram conduzidos independentemente, em casa de vegetação do Departamento de Proteção Vegetal da Faculdade de Ciências Agronômicas, FCA/UNESP, Câmpus de Botucatu-SP.

Nos estudos com $M$. incognita e $M$. enterolobii, os genótipos avaliados foram: 'White Adriatic', 'Caprifigo IAC', 'Celeste IAC', 'Genoveso IAC' e 'Roxo de Valinhos'. Os mesmo genótipos, mais 'Stanford IAC', foram testados também frente a M. javanica. Tais genótipos foram obtidos a partir de estacas procedentes da Coleção do Banco de Germoplasma de Figo do Departamento de Fitotecnia, Tecnologia de Alimentos e Socioeconomia, da Faculdade de Engenharia de Ilha Solteira - FEIS/ UNESP, Câmpus de Ilha Solteira-SP.

Estacas com aproximadamente $20 \mathrm{~cm}$ de comprimento por $1,5 \mathrm{~cm}$ de diâmetro foram cortadas em bisel e plantadas em vasos de 5L contendo areia autoclavada. Após o enraizamento, as mudas foram transplantadas para sacos plásticos contendo $2 \mathrm{~L}$ de substrato composto por terra, areia e matéria orgânica (1:2:1), previamente autoclavados.

A população de $M$. javanica foi obtida de raízes de tomateiro 'Magali' provenientes do município de Santa Rosa-RS. A de M. incognita raça 2, por sua vez, foi obtido de raízes de cafeeiro provenientes do município de Oswaldo Cruz-SP., e a população de M. enterolobii foi obtida a partir de pimentão 'Silver' em Campos Novos Paulista-SP. As espécies foram identificadas pelo padrão perineal das fêmeas, segundo técnica de Taylor e Netscher (1974) e pelo padrão eletroforético de isoenzimas, conforme Esbenshade e Triantaphyllou (1990) e têm sido mantidas em tomateiros 'Rutgers' na casa de vegetação do Departamento de Proteção Vegetal.

Raízes infectadas com cada uma das espécies estudadas foram processadas separadamente, seguindo a metodologia proposta por Hussey e Baker (1973), modificada por Bonetti e Ferraz (1981), a qual consiste na trituração destas raízes infectadas em liquidificador com solução de hipoclorito de sódio a $0,5 \%$, seguida de peneiramento. Os ovos obtidos foram contados em lâmina de Peters, sob microscópio fotônico. A suspensão foi calibrada para 2.500 ovos $/ \mathrm{mL}$.

O substrato de cada parcela foi infestado com 5.000 ovos, adicionando-se $2 \mathrm{~mL}$ da suspensão no interior de dois orifícios com aproximadamente dois centímetros de profundidade, sobre as raízes.

O delineamento experimental utilizado foi o inteiramente ao acaso, com cinco repetições, sendo cada parcela constituída por uma planta por vaso. Tomateiros 'Rutgers' foram utilizados como padrão de viabilidade de inóculo em todos os experimentos, contudo foram avaliados antes da finalização do experimento, aos 60 dias.

As avaliações foram feitas aos 120 dias após a inoculação, em todos os experimentos. Para a coloração das massas de ovos externas dos nematoides, os sistemas radiculares foram lavados em água corrente e, em seguida, submetidos à coloração com Floxina B, como descrito por Taylor e Sasser (1978). Os índices de galhas (IG) e de massas de ovos (IMO) foram obtidos de acordo com a escala de notas proposta pelos mesmos autores em que: $0=$ sem galhas ou massas de ovos; $1=1$ a 2 ; $2=3$ a $10 ; 3=11$ a $30 ; 4=31$ a 100 , e $5=$ mais de 100 galhas ou massas de ovos por raiz. Em seguida, os sistemas radiculares foram processados segundo o método de Coolen e D'Herde (1972), usando-se solução de hipoclorito de sódio a $0,5 \%$ no lugar da água, para triturar as raízes no liquidificador com posterior centrifugação para a obtenção da suspensão. 
Para a determinação do número de ovos e dos eventuais juvenis de segundo estádio, a suspensão ao final do experimento (Pf) foi efetuada com o auxílio da lâmina de Peters, em microscópio fotônico. Tal valor foi utilizado para a obtenção do fator de reprodução $(\mathrm{FR}=\mathrm{Pf} /$ População inicial $(\mathrm{Pi}))$ de cada nematoide, em cada genótipo estudado. As plantas com $\mathrm{FR}<1$ foram consideradas resistentes, e com FR $>1$ ou igual a 1 , suscetíveis (OOSTENBRINK, 1966). Entretanto, para a verificação da variação na resistência entre genótipos, os dados do FR foram transformados em $\sqrt{ } \mathrm{x}+0,5$ e submetidos à análise de variância, sendo as médias comparadas pelo teste de Scott-knott, a 5\% de probabilidade, com auxílio do programa computacional Sisvar versão 5.3 (COOK; STARR 2006; FERREIRA, 2010).

\section{RESULTADOS E DISCUSSÃO}

Meloidogyne incognita conseguiu multiplicarse em todos os genótipos de figo estudados, com FR variando de 8,5 para 'Genoveso IAC' a 25,9 para 'White Adriatic'. Os genótipos 'White Adriatic' e 'Roxo de Valinhos' não diferiram estatisticamente entre si, mas apresentaram maiores FRs quando comparados com os outros genótipos. Os IGs e os IMOs variaram de 2,6 a 4,0, e de 2,4 a 4,0 respectivamente (Tabela1).

Não houve diferenças significativas quanto ao FR de $M$. enterolobii nos genótipos estudados, variando de 6,6 para 'Celeste IAC' a 10,3 para 'Genoveso IAC' (Tabela 2). Os IGs variaram de 3,8 a 5,0 para 'White Adriatic' e 'Roxo de Valinhos', respectivamente. Os IMOs variaram de 3,6 para 'White Adriatic' a 4,2 para 'Genoveso IAC', 'Caprifigo IAC' e 'Celeste IAC'.

Também não houve diferenças no FR de $M$. javanica nos genótipos de figo estudados, variando de 3,4 para 'Celeste IAC' a 7,2 para 'Roxo de Valinhos' (Tabela 3). Os IGs variaram de 2,0 para 'Genoveso IAC' a 2,4 para 'Roxo de Valinhos', 'White Adriatic' e 'Celeste IAC'. Os IMOs variaram de 1,0 para 'Caprifigo IAC' a 2,0 para 'Stanford IAC' e 'Celeste IAC'.

Em todos os experimentos, a viabilidade dos inóculos dos diferentes nematoides foi confirmada pelo FR maior que um em plantas de tomate.

Embora a suscetibilidade de $F$. carica aos nematoides-das-galhas tenha sido relatada, pouco se conhece sobre suas variações nos diferentes genótipos. A resistência a esses nematoides já foi detectada na Califórnia, em outras quatro espécies de Ficus; F. racemosa L., F. cocculifolia Baker, F. gnaphalocarpa Steud. ex. Miquel, e F. palmata
Forsk (EL- BORAI; DUNCAN, 2005). Também no Japão, experimentos conduzidos em vasos e em campo demonstraram a tolerância da cultivar Zidi enxertada no porta-enxerto 'Masui Dauphine' a $M$. incognita. Entretanto, em ambos os experimentos, as populações iniciais do nematoide eram desconhecidas (HOSOMI et al., 2002).

A cultivar Celeste é tida como resistente a Meloidogyne spp. em estudos desenvolvidos em Israel (GUR, 1955; EL-BORAI; DUNCAN, 2005). Entretanto, no presente estudo, a cultivar Celeste comportou-se como suscetível às três espécies de Meloidogyne testadas. Isto pode ter ocorrido devido ao fato de as cultivares, embora com a mesma denominação, serem distintas, por se tratar de experimentos conduzidos em diferentes países e em épocas distantes ou tratar-se de raças diferentes do nematoide.

Os IGs e IMOs para M.javanica apresentaramse relativamente baixos; no entanto, o FR foi acima de um em todos os genótipos, comprovando sua capacidade de multiplicação. Isso se deve, provavelmente, devido ao desenvolvimento das massas de ovos internamente nas raízes, uma vez que nematoides do gênero Meloidogyne podem deixar suas massas de ovos externamente às raízes ou internamente, isso depende, na maioria das vezes, da reação do hospedeiro. O mesmo ocorre com as galhas, sendo estas reações da planta ao parasitismo do nematoide (KARSSEN, MOENS, 2006). Os valores baixos de IG indicam que os genótipos de figo ora estudados não permitem a formação de galhas bem definidas quando parasitados por $M$. javanica. Os dados de FR para o figo conduzem a inferir que a espécie $M$. javanica se apresentou com o menor valor entre as espécies de nematoides avaliadas.

No Brasil, a suscetibilidade a $M$. incognita da cultivar Roxo de Valinhos foi verificada juntamente com outras três cultivares (MOURA, 1967). A partir daí, nenhum outro estudo nematológico testando a resistência desta planta foi desenvolvido no País, que confirmou a suscetibilidade desta cultivar a este nematoide e a outras duas espécies, M. enterolobii e M. javanica.

Apesar de M. enterolobii não ter sido relatada em pomares de figo até o momento, sua multiplicação nos genótipos testados, inclusive na cultivar Roxo de Valinhos, traz preocupação, uma vez que esta espécie de nematoide-das-galhas tem-se tornado fator limitante para a produção de outras frutíferas, como a goiabeira, na qual foi detectada pela primeira vez em Petrolina-PE, Curaçá e Maniçoba-BA, sendo causadora da morte de muitos pomares desta frutífera devido à associação com Fusarium solani (Mart.) 
Sacc. (CARNEIRO et al., 2001 ; GOMES et al., 2012). Medidas de prevenção devem ser tomadas para que este nematoide não se torne problema também nesta cultura.

Embora tenham sido observadas variações nos FRs das três espécies de Meloidogyne estudadas nos diferentes genótipos, a suscetibilidade dos mesmos impede-os de serem utilizados como porta-enxerto, uma vez que mesmo nos casos em que os genótipos apresentaram FR mais baixo que os demais, sendo este acima de um, permite a multiplicação do patógeno. Tratando-se de uma cultura perene, a população poderá atingir níveis elevados, sendo esse problema agravado pela falta de opção de controle e pela ausência de cultivares ou porta-enxertos resistentes aos nematoides. Sendo assim, medidas preventivas devem ser adotadas a fim de evitar a introdução desses patógenos na área, principalmente utilizando mudas isentas.

TABELA 1- Valores médios do índice de galhas (IG), índice de massas de ovos (IMO) e fator de reprodução (FR) por sistema radicular, em genótipos de figueiras inoculados com 5.000 ovos de Meloidogyne incognita.

\begin{tabular}{lcccc}
\hline Genótipo & IG & IMO & FR* $^{*}$ & Reação \\
\hline White Adriatic & 4,0 & 4,0 & $25,9 \mathrm{a}$ & $\mathrm{S}$ \\
Roxo de Valinhos & 4,0 & 4,0 & $20,3 \mathrm{a}$ & $\mathrm{S}$ \\
Caprifigo IAC & 3,0 & 2,8 & $14,9 \mathrm{~b}$ & $\mathrm{~S}$ \\
Celeste IAC & 3,8 & 3,0 & $10,7 \mathrm{~b}$ & $\mathrm{~S}$ \\
Genoveso IAC & 2,6 & 2,4 & $8,5 \mathrm{~b}$ & $\mathrm{~S}$ \\
\hline CV (\%) & & & & 21,3 \\
'Rutgers' & 4,8 & 5,0 & 35,6 & \\
\hline
\end{tabular}

Médias na coluna seguidas pela mesma letra não diferem entre si, pelo teste de Scott-knott, a $5 \%$ de probabilidade. *Análise realizada a partir de dados transformados em $\sqrt{X}_{\mathrm{X}}+0,5 . \mathrm{R}=$ resistente $(\mathrm{FR}<1,0)$ e $\mathrm{S}=$ suscetível (FR $\left.\geq 1,0\right)$, segundo OOSTENBRINK, 1966.

TABELA 2- Valores médios do índice de galhas (IG), índice de massas de ovos (IMO) e fator de reprodução (FR) por sistema radicular, em genótipos de figueiras inoculados com 5.000 ovos de Meloidogyne enterolobii.

\begin{tabular}{lcccc}
\hline Genótipo & IG & IMO & FR* $^{*}$ & Reação \\
\hline Genoveso IAC & 4,8 & 4,2 & $10,3^{\text {ns }}$ & $\mathrm{S}$ \\
Roxo de Valinhos & 5,0 & 4,0 & 9,9 & $\mathrm{~S}$ \\
White Adriatic & 3,8 & 3,6 & 8,8 & $\mathrm{~S}$ \\
Caprifigo IAC & 4,4 & 4,2 & 8,4 & $\mathrm{~S}$ \\
Celeste IAC & 4,4 & 4,2 & 6,6 & $\mathrm{~S}$ \\
\hline CV (\%) & 5,0 & 5,0 & 28,5 & 29,8 \\
'Rutgers' & &
\end{tabular}

ns Não significativo pelo teste $\mathrm{F}$, a $5 \%$ de probabilidade. *Análise realizada a partir de dados transformados em $\sqrt{ } \mathrm{X}+0,5$. $\mathrm{R}=$ resistente (FR $<1,0)$ e $\mathrm{S}=$ suscetível $(\mathrm{FR} \geq 1,0)$, segundo Oostenbrink, (1966).

TABELA 3- Valores médios do índice de galhas (IG), índice de massas de ovos (IMO) e fator de reprodução (FR) por sistema radicular, em genótipos de figueiras inoculados com 5.000 ovos de Meloidogyne javanica.

\begin{tabular}{ccccc}
\hline Genótipo & IG & IMO & FR* $^{*}$ & Reação \\
\hline Roxo de Valinhos & 2,4 & 1,8 & $7,2^{\text {ns }}$ & $\mathrm{S}$ \\
Caprifigo IAC & 2,2 & 1,0 & 6,1 & $\mathrm{~S}$ \\
Stanford IAC & 2,2 & 2,0 & 4,1 & $\mathrm{~S}$ \\
Genoveso IAC & 2,0 & 1,4 & 4,0 & $\mathrm{~S}$ \\
White Adriatic & 2,4 & 1,6 & 3,5 & $\mathrm{~S}$ \\
Celeste IAC & 2,4 & 2,0 & 3,4 & $\mathrm{~S}$ \\
\hline CV (\%) & & & & 33,7 \\
'Rutgers' & 5,0 & 5,0 & 33,7 & \\
\hline
\end{tabular}

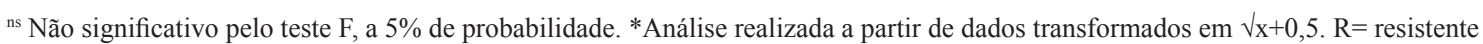
(FR $<1,0)$ e $\mathrm{S}=$ suscetível (FR $\geq 1,0)$, segundo Oostenbrink, (1966). 


\section{CONCLUSÃO}

Os genótipos de figueira estudados não são indicados para plantio em áreas infectadas $\operatorname{com} M$. javanica, $M$. incognita e $M$. enterolobii, uma vez que os mesmos permitiram a multiplicação do nematoide.

\section{REFERÊNCIAS}

ABRANTES, I.M.O.;VIERA DOS SANTOS, M.C.; CONCEIÇÃO, I.L.P. M.; SANTOS, M.S.N.A.; VOVLAS, N. Root-knot and other plant-parasitic nematodes associated with fig trees in Portugal. Nematologia Mediterranea, Bari, v.36, p.131-136, 2008.

AGROFIT: Sistema de agrotóxicos fitossanitários. Disponível em: <http://extranet.agricultura.gov.br/ agrofit_cons/principal agrofit_cons $>$. Acesso em: 15 fev. 2014.

BONETI, J.I.S.; FERRAZ, S. Modificação do método de Hussey e Barker para extração de ovos de Meloidogyne exigua de cafeeiro. Fitopatologia Brasileira, Brasília, v.6, n.3, p.553, 1981.

CAMPOS, V.P. Nematoides na cultura da figueira. Informe Agropecuário, Belo Horizonte, v.18, n.1, p.33-38, 1997.

CARNEIRO, R.M.D.G.; MOREIRA, W.A.; ALMEIDA, M.R.A.; GOMES, A.C.M.M. Primeiro registro de Meloidogyne mayaguensis em goiabeira no Brasil. Nematologia Brasileira, Brasília, v.25, n.2, p.223-228, 2001.

COOK, R.; STAR, J.L. Resistant cultivars. In: PERRY, R.N.; MOENS, M. Plant nematology. Wallingford: CAB International, 2006. p.370- 391.

COOLEN, W.A; D'HERDE, C.J. A method for quantitative extraction of nematodes from plant tissue. Ghent: State Nematology end Entomology Research Station, 1972. p.77.

DIAS-ARIEIRA, C.R.; FURLANETTO, C.; SANTANA, S.M.; BARIZÃO, D.A.O.; RIBEIRO, R.C.F.; FORMENTINI, H.M. Fitonematoides associados a frutíferas na região noroeste do Paraná, Brasil. Revista Brasileira de Fruticultura, Jaboticabal, v.32, n.4, p.1064-1071, 2010.
EL-BORAI, F.E.; DUNCAN, L. W. Nematode parasites of subtropical and tropical fruit tree crops. In: LUC, M.; SIKORA, R.A.; BRIDGE, J. Plant parasitic nematodes in subtropical and tropical agriculture. $2^{\text {nd }} \mathrm{ed}$. Washington: $\mathrm{CAB}$ International, 2005. p.467-492.

ESBENSHADE, P.R.; TRIANTAPHYLLOU, A.C. Isozyme phenotypes for the identification of Meloidogyne species. Journal of Nematology, Lawrence, v.22, n.1, p.10-15, 1990.

FAO - Food Agricultural Organization. Top producción-higos. 2012. Disponível em: <http:// faostat.fao.org. . . Acesso em: 2 nov. 2013.

FERREIRA, D.F. Sisvar 5.3. Sistema de análises estatísticas. Lavras: UFLA, 2010.

GOMES, V.M.; SOUZA, R.M.; MIDORIKAWA, G.; MILLER, R.; ALMEIDA, A.M. Guava decline: evidence of nationwide incidence in Brazil. Nematropica, Gainesville, v.42, n.1, p.153-162, 2012.

GUR, A. The fig. Israel: Departments of Horticulture and Agricultural Education, Ministry of Agriculture, 1955. (Bulletin, 57).

HOSOMI, A.; DAN, M.; KATO, A. Screening of fig varieties for rootstocks resistant to soil sickness. Journal of the Japanese Society for Horticultural Science, Tokyo, v.71, n.2, p.171-176, 2002.

KARSSEN, G.; MOENS, M. Root-knot Nematodes. In: PERRY, R.N.; MOENS, M. Plant nematology. Wallingford: CABI, 2006. p.59-88.

LEONEL, S. A figueira. Revista Brasileira de Fruticultura, Jaboticabal, v.30, n.3, p. 577-856, 2008.

LIMA-MEDINA, I.; GOMES, C.B.; ROSSI, C.; CARNEIRO, R.M.D.G. Caracterização e identificação de populações de nematoides de galhas provenientes de figueiras (Ficus carica L.) do Rio Grande do Sul e de São Paulo. Nematologia Brasileira, Brasília, v.30, n.2, p.179-187, 2006.

LIMA-MEDINA, I.; SOMAVILLA, L.; CARNEIRO, R.M.D.G.; GOMES, C.B. Espécies de Meloidogyne em figueira (Ficus carica) e em plantas infestantes. Nematropica, Gainesville, v.43, n.1, p56-62, 2013. 
MCSORLEY, R. Nematological problems in tropical and subtropical fruit tree crops. Nematropica, Gainesville, v.22, n.1, p.103-116, 1992.

MOURA, R.M. Contribuição ao estudo da meloidoginose da figueira (Ficus carica L.). 1967. 28f. Mestrado (Magister Scientiae) - Escola Superior de Agricultura Luiz de Queiroz, Universidade de São Paulo, Piracicaba, 1967.

NEAL, J.C. The root-knot disease of the peach, orange and other plants in Florida due to the work of Anguillula. Bulletin of the United States Bureau of Entomology, Washington, v.20, p.1-31, 1889.

OOSTENBRINK, M. Major characteristics of the relation between nematodes and plants. Mededelingen, Wageningen, v.66, p.1-46, 1966.

PENTEADO, S.R. O cultivo da figueira no Brasil e no Mundo. In: CORRÊA, L.S.; BOLIANI, A.C. Cultura da figueira: do plantio à comercialização. Ilha Solteira: FAPESP, 1999. p.1-16.
PIO, R.; LEONEL, S.; CHAGAS, E.A. Aspectos botânicos e biologia reprodutiva da figueira. In: LEONEL. S.; SAMPAIO, A.C. A figueira. São Paulo: Editora UNESP, 2011. p. 67-76.

SILVA, A.C.; VASONCELLOS, M.A.S.; BUSQUET, R.N.B. Aspectos econômicos da produção e comercialização do figo. In: LEONEL. S.; SAMPAIO. A.C. A figueira. São Paulo: Editora UNESP, 2011. p.57-66.

TAYLOR, A.L.; SASSER, J.N. Biology, identification and control of root-knot nematodes (Meloidogyne species). Raleigh: North Caroline State University, 1978. 111p.

TAYLOR, D.P.; NETSCHER, C. An improved technique for preparing perineal patterns of Meloidogyne spp. Nematropica, Gainesville, v.20, p.268. 1974.

WOHLFARTER, M.; GILIOMEE, J.H.; VENTER, E.; STOREY, S. A Survey of the Arthropod Pests and Plant Parasitic Nematodes Associated with Commercial Figs, Ficus carica (Moraceae), in South Africa. African Entomology, Johannesberg, v.19, n.1, p.165-172, 2011. 